EPJ Web of Conferences 66, 04002 (2014)

DOI: $10.1051 /$ epjconf/ 20146604002

(C) Owned by the authors, published by EDP Sciences, 2014

\title{
Strange hadrons and resonances at LHC energies with the ALICE detector
}

\author{
A. Badalá ${ }^{1, a}$ for the ALICE Collaboration \\ ${ }^{1}$ INFN-Sezione di Catania, V. S. Sofia 64, Catania, 195125, Italy
}

\begin{abstract}
The study of (multi)strange hadrons and resonance production allows in heavyion collisions to gather information on the early partonic phase of the fireball and its evolution. The ALICE collaboration has measured the production of $\mathrm{K}^{*}(892)^{0}, \phi(1020)$ resonances and $\mathrm{K}_{\mathrm{S}}^{0}, \Lambda, \Xi^{-}, \Omega^{-}$hadrons and their anti-particles at mid-rapidity in $\mathrm{Pb}-\mathrm{Pb}$ collisions at $\sqrt{s_{\mathrm{NN}}}=2.76 \mathrm{TeV}$. This contribution presents results on strangeness enhancement, baryon to meson ratios $\left(\Lambda / \mathrm{K}_{\mathrm{S}}^{0}\right.$ and $\left.\Omega^{-} / \phi\right)$ and on resonance to stable particle ratio $(\phi / \mathrm{K}$ and $\left.\mathrm{K}^{*} / \mathrm{K}\right)$. The nuclear modification factor $R_{\mathrm{AA}}$ of the $\phi(1020)$ will be also discussed.
\end{abstract}

\section{Introduction}

Strange hadrons and resonances are sensitive probes to investigate the characteristics of the fireball formed in ultrarelativistic heavy-ion collisions. In particular, hadronic resonances probe the dynamical evolution of the fireball. Due to their short lifetime (a few $\mathrm{fm} / \mathrm{c}$ ) a significant fraction decays during the evolution from chemical to kinetic freeze-out. Products of their hadronic decay may rescatter reducing the measured resonance signal. Resonance may also be regenerated through collisions of hadrons. The competition between regeneration and rescattering effects could be used to estimate the timescale between chemical and kinetic freeze-out [1]. The results reported in the following paragraphs refer to analyses carried out on minumum-bias $\mathrm{Pb}-\mathrm{Pb}$ data at $\sqrt{s_{\mathrm{NN}}}=2.76 \mathrm{TeV}$ collected during 2010. The tracking detectors used for these analyses are the Inner Tracking System (ITS) and the Time Projection Chamber (TPC), which also identifies particles via their specific energy loss. Strange hadrons and resonances were identified by reconstructing the following decays (and their charge conjiugate, where relevant) into charged particles: $\mathrm{K}_{\mathrm{S}}^{0} \rightarrow \pi^{+}+\pi^{-}, \Lambda \rightarrow \mathrm{p}+\pi^{-}, \Xi^{-} \rightarrow \Lambda+\pi^{-}$, $\Omega^{-} \rightarrow \Lambda+\mathrm{K}^{-}, \mathrm{K}^{*}(892)^{0} \rightarrow \pi^{+}+\mathrm{K}^{-}, \phi(1020) \rightarrow \mathrm{K}^{+}+\mathrm{K}^{-}$. Daughter tracks were identified by their specific energy loss in the TPC. Strange and multi-strange hadrons were reconstructed exploting their $\mathrm{V}^{0}$ and cascade topology. Due to the short lifetime of the resonances their yield is obtained by computing the invariant mass spectrum with all combinations of primary tracks and then subtracting the combinatorial background. This was estimated using the event-mixing or the like-sign technique. The residual background was fitted by a polynomial.

\section{Strangeness enhancement}

An enhanced production of strange particles in ultrarelativistic heavy-ion collisions with respect to elementary collisions is one of the historical signatures [2] of the quark-gluon-plasma formation.

a. e-mail: Angela.Badala@ct.infn.it

This is an Open Access article distributed under the terms of the Creative Commons Attribution License 2.0, which permits unrestricted use, distribution, and reproduction in any medium, provided the original work is properly cited. 

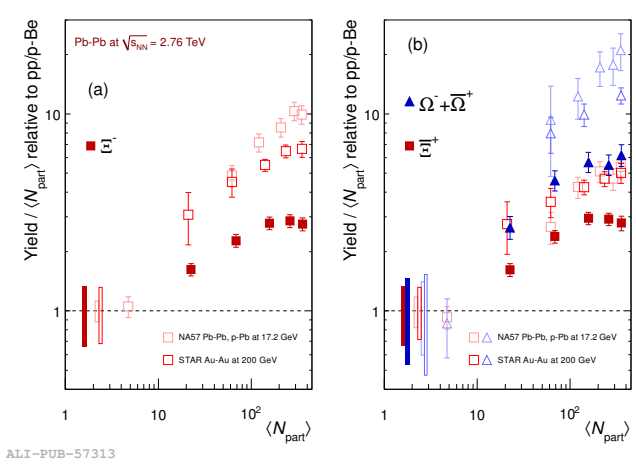

Figure 1. Strangeness enhancement of $\Xi^{-}, \bar{\Xi}^{+}$, $\Omega^{-}+\bar{\Omega}^{+}$as a function of the mean number of participants from LHC (ALICE Pb-Pb at the $\sqrt{s_{\mathrm{NN}}}=2.76 \mathrm{TeV}$, full symbols), RHIC and SPS (open symbols) data. The LHC data use interpolated pp values, from lower [3] and higher [4] collision energy pp data. The enhancements at lower energy were measured from NA57 [5] and STAR [6] in Pb-Pb (normalized to p-Be collisions) and $\mathrm{Au}-\mathrm{Au}$ (normalized to pp collisions) collisions, respectively.

The idea was that in a deconfined system of quarks and gluons s̄̄ pairs are more easily produced than strange-antistrange hadron pairs in a hadron gas and the time to reach equilibrium is also much shorter in the first case. The strangeness enhancement is defined as the ratio of the yield of strange hadrons to the same yield in elementary collisions, both normalized to the number of participating nucleons. This is expected to be equal to one if the strange particle production scales with the number of participating nucleons. An increase with the centrality of the collisions and with the strangeness content of the particle was expected. In fig. 1 the strangeness enhancement of $\Xi^{-}, \bar{\Xi}^{+}, \Omega^{-}+\bar{\Omega}^{+}$measured by ALICE in $\mathrm{Pb}-\mathrm{Pb}$ collisions at $\sqrt{s_{\mathrm{NN}}}=2.76 \mathrm{TeV}$ as a function of the mean number of participant nucleons is shown [7]. The enhancements measured at lower energies are also shown. One observes that this ratio is larger than one and increases with the centrality, and that there is a hierarchy in the production based on the strangeness content of the particle. However this ratio decreases with the collision energy. Canonical suppression [8] qualitatively describes the trend of strangeness yield with centrality and energy: the production of strange particles in pp increases with energy thus reducing the enhancements.

\section{Resonance to stable particle ratios and baryon to meson ratios}

While the $\phi / \mathrm{K}$ ratio is independent of the collision centrality, the $\mathrm{K}^{*} / \mathrm{K}$ ratio shows a hint of a decrease increasing the centrality (fig. 2). In addition the $\phi / \mathrm{K}$ ratio is independent of the collision energy and of the collision system and it is in agreement with thermal model prediction [9], which, on the contrary, clearly overpredict the $\mathrm{K}^{*} / \mathrm{K}$ ratio. Considering the factor of about 10 between the lifetimes of the two resonances, the origin of the differences in the $\mathrm{K}^{*}$ and $\phi$ production could be related to a large modification of the $\mathrm{K}^{*}$ yield due to the final-state interactions (rescattering and regeneration).

In fig. 3 the $\Lambda / \mathrm{K}_{\mathrm{S}}^{0}$ ratio as a function of $p_{\mathrm{T}}$ is shown for various centrality classes of $\mathrm{Pb}-\mathrm{Pb}$ collisions at $\sqrt{s_{\mathrm{NN}}}=2.76 \mathrm{TeV}$ [10] and compared to the same ratio in pp collisions at the energy of $\sqrt{s}=0.9$ and $7 \mathrm{TeV}$. One can observe that the production of baryons excedes that of mesons in central collisions at intermediate transverse momentum $\left(3<p_{\mathrm{T}}<5 \mathrm{GeV} / c\right)$. The baryon/meson ratio is similar in $\mathrm{pp}$ and in peripheral $\mathrm{Pb}-\mathrm{Pb}$ collisions and increases with centrality, exceding unity in the most central collisions. The peak is shifted towards high transverse momentum going from peripheral to central collisions. One possible explanation of the so-called "baryon anomaly", alredy observed at RHIC energies, is the recombination of quarks (coalescence) as an additional hadronization mechanism [11] in presence of a deconfined medium. In general, the interplay of effects as flow, recombination and fragmentation has to be taken into account when modeling the intermediate $p_{\mathrm{T}}$ region in ultrarelativistic heavy-ion collisions. The mechanisms of strange and multi-strange particle production are sensitive to 


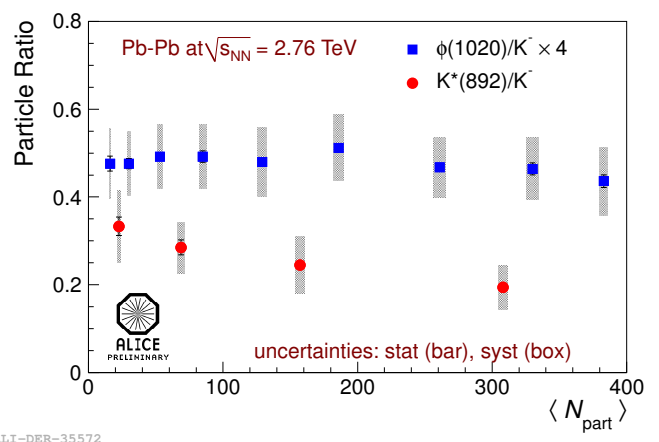

Figure 2. $\mathrm{K}^{*} / \mathrm{K}$ and $\phi / \mathrm{K}$ ratios as a function of the mean number of participants in $\mathrm{Pb}-\mathrm{Pb}$ collisions at $\sqrt{s_{\mathrm{NN}}}=2.76 \mathrm{TeV}$.

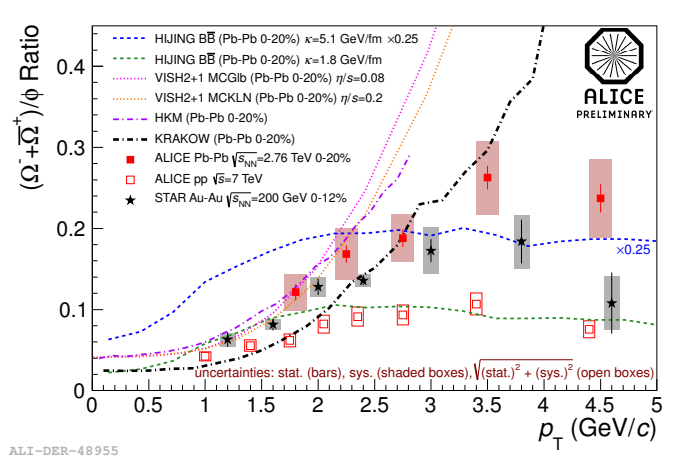

Figure 4. $\left(\Omega^{-}+\bar{\Omega}^{+}\right) / \phi$ as a function of $p_{\mathrm{T}}$ for $\mathrm{Pb}-\mathrm{Pb}$ collisions at $\sqrt{s_{\mathrm{NN}}}=2.76 \mathrm{TeV}$ in the centrality bin $0-20 \%$. STAR data [16] for Au-Au collisions at $\sqrt{s_{\mathrm{NN}}}=200 \mathrm{GeV}$ (star symbol) and ALICE data(open symbol) for pp collisions at $\sqrt{s}=7 \mathrm{TeV}$ [17] are also shown. Curves represent predictions of different models (see text).

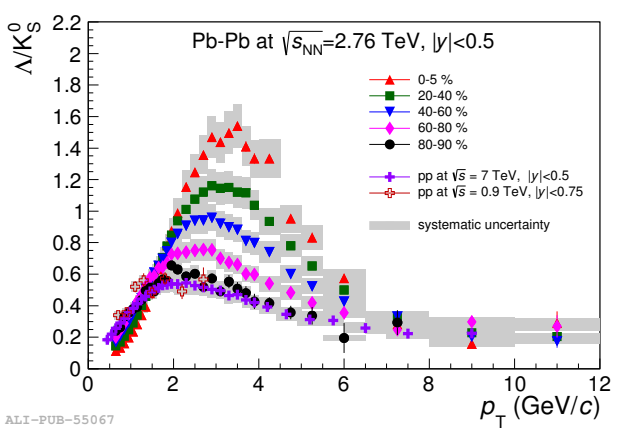

Figure 3. $\Lambda / \mathrm{K}_{\mathrm{S}}^{0}$ ratio as a function of $p_{\mathrm{T}}$ for several centrality classes in $\mathrm{Pb}-\mathrm{Pb}$ collisions at $\sqrt{s_{\mathrm{NN}}}=2.76 \mathrm{TeV}$ and for pp collisions at $\sqrt{s}=0.9$ and $7 \mathrm{TeV}$.

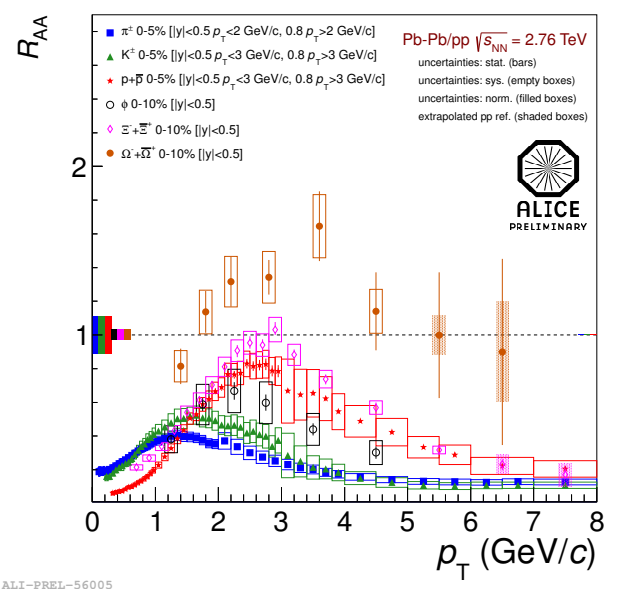

Figure 5. Nuclear modification factor of $\pi^{ \pm}, \mathrm{K}^{ \pm}$, $\mathrm{p}+\overline{\mathrm{p}}$ and $\phi, \Xi^{-}+\bar{\Xi}^{+}, \Omega^{-}+\bar{\Omega}^{+}$in $\mathrm{Pb}-\mathrm{Pb}$ collisions at $\sqrt{s_{\mathrm{NN}}}=2.76 \mathrm{TeV}$ in the centrality bins $0-5 \%$ and 0 $10 \%$, respectively.

the early phase of the nuclear collisions. In fig. 4 the $\left(\Omega^{-}+\bar{\Omega}^{+}\right) / \phi$ ratio as a function of the transverse momentum is shown. The ratios measured by ALICE in $\mathrm{Pb}-\mathrm{Pb}$ collisions at $\sqrt{s_{\mathrm{NN}}}=2.76 \mathrm{TeV}$ and in pp collisions at $7 \mathrm{TeV}$ are compared with STAR data for Au-Au collisions at $\sqrt{s_{\mathrm{NN}}}=200 \mathrm{GeV}$ [16] and with the predictions of different models. The ratios measured at LHC energy are similar to the ones obtained at RHIC energies. The HKM and VISH2+1 hydrodynamical models $[12,13]$ are able to reproduce low $p_{\mathrm{T}}$ data, while the KRAKOW [14] model largely underprects the ratio. The expected large string value $(\mathrm{k}=5 \mathrm{GeV} / \mathrm{fm})$ predicted from the HIJING BB model [15], in which particle production is modeled by a Strong Color Field, largely overpredicts the experimental data. However the same model was able to reproduce the same ratio in Au-Au collisions at $\sqrt{s_{\mathrm{NN}}}=200 \mathrm{GeV}$ [16] and in pp collisions at $7 \mathrm{TeV}$ [17]. 


\section{Nuclear modification factor}

In central AA collisions a suppression of the production of high $p_{\mathrm{T}}$ particles has been observed alredy at RHIC energies, consistent with the formation of a coloured, dense fireball. A larger suppression has been reported by the ALICE collaboration [18]. To quantify nuclear medium effect at high $p_{\mathrm{T}}$, the so-called modification factor $R_{\mathrm{AA}}$ is used. It is defined as $R_{\mathrm{AA}}\left(p_{\mathrm{T}}\right)=\frac{\mathrm{d} N_{\mathrm{AA}} / \mathrm{d} p_{\mathrm{T}}}{\left\langle T_{\mathrm{AA}}\right\rangle \mathrm{d} \sigma_{\mathrm{pp}} / \mathrm{d} p_{\mathrm{T}}}$, where $N_{\mathrm{AA}}$ and $\sigma_{\mathrm{pp}}$ represent the charged particle yield in nucleus-nucleus collisions and the cross section in $\mathrm{pp}$ collisions, respectively. $T_{\mathrm{AA}}$ is the nuclear overlap function, computed in the framework of a Glauber model [19]. In central collisions no strong flavour or baryon/meson dependence is observed in the $R_{\mathrm{AA}}$ of $\pi, \mathrm{K}$ and p of $p_{\mathrm{T}}>8 \mathrm{GeV} / c$. On the contrary a large baryon/meson depence is observed for low $p_{\mathrm{T}}$ (fig. 5) in particular $R_{\mathrm{AA}}(\mathrm{p})>R_{\mathrm{AA}}(\pi)$ and the $R_{\mathrm{AA}}$ of the $\phi$ is slightly larger than that of $\pi$.

\section{Conclusions}

Some of the main results on strange particle production obtained by the ALICE collaboration in $\mathrm{Pb}-\mathrm{Pb}$ collisions at $\sqrt{s_{\mathrm{NN}}}=2.76 \mathrm{TeV}$ have been presented. The data are consistent with the creation of a hot, dense and coloured fireball with a large radial flow. In particular, a large baryon/meson depencence is observed in central collisions at low $p_{\mathrm{T}}$, where the $R_{\mathrm{AA}}$ (baryons) $>R_{\mathrm{AA}}$ (mesons) and the $\Lambda / \mathrm{K}_{\mathrm{S}}^{0}$ ratio is larger than one. The $\mathrm{K}^{*} / \mathrm{K}$ ratio shows a hint of decrease with increasing centrality, while the $\phi / \mathrm{K}$ ratio is independent of centrality. This could be due to the interaction with the hadronic medium of the $\mathrm{K}^{*}$ daughters and could be used to estimate the time span between the chemical and the kinetic freeze-out.

\section{References}

[1] G. Torrieri and J. Rafelski, J. Phys. G28, 1911 (2002); C. Markert et al., hep-ph/0206260.

[2] J. Rafelski and B. Muller, Phys. Rep. 142, 167 (1982); P. Koch et al., Phys. Rep. 142, 167 (1986)

[3] K. Aamodt et al. (ALICE Coll.), Eur. Phys. J. C71, 1594 (2011); B.I. Abelev et al. (STAR Coll.), Phys. Rev. C75, 064901 (2007)

[4] B. Abelev et al. (ALICE Coll.), Phys. Lett. B712, 309 (2012)

[5] F. Antinori et al. (NA57 Coll.), J. Phys. G37, 045105 (2010)

[6] G. Agakishiev et al. (STAR Coll.), Phys. Rev. Lett. 106, 032301 (2011)

[7] B. Abelev et al. (ALICE Coll.), arXiv:1307.5543

[8] S. Hamieh et al., Phys. Lett. B486, 61 (2000); A. Tounsi et al., arXiv:0111159v1

[9] A. Andronic et al., Phys. Lett. B673, 142 (2009); Acta Phys. Pol. 40, 1005 (2009)

[10] B. Abelev et al. (ALICE Coll.), arXiv:1307:5530

[11] R. Fries et al., Ann. Rev. Nucl. Part. Sci. 58, 177 (2008)

[12] I. Karpenko et al., Phys. Rev. C87, 024914 (2013)

[13] C. Shen et al., Phys. Rev. C84, 044903 (2011); Z. Qiu et al, Phys. Lett. B707, 151 (2012)

[14] P. Bozek et al., Phys. Rev. C85, 064915 (2012)

[15] V. Topor Pop et al., Phys. ReV. C84, 044909 (2011)

[16] B.I. Abelev et al. (STAR Coll.), Phys. Rev. C79, 06903 (2009)

[17] B. Abelev et al. (ALICE Coll.), Eur. Phys. J. C72, 2183 (2012)

[18] K. Aamodt et al. (ALICE Coll.), Phys. Lett. B696, 30 (2011); Abelev et al. (ALICE Coll.), Phys. Lett. B720, 52 (2013)

[19] M. Miller et al., Ann. Rev. Nucl. Part. Sci. 57, 205 (2007) 